\title{
Rotary slanted single wire CTA - a useful tool for 3D flows investigations
}

\author{
P. Jonáś* \\ ${ }^{1}$ Institute of Thermomechanics Academy of Sciences of the Czech Republic, v.v.i., Praha, Czech Republic
}

\begin{abstract}
The procedure is described of experimental investigation of a statistically stationary generally nonisothermal 3D flow by means of a constant temperature anemometer (CTA) using single slanted heated wire, rotary round the fixed axis. The principle of this procedure is quite clear. The change of the heated wire temperature modifies ratio of CTA sensitivities to temperature and velocity fluctuations. Turning the heated wire through a proper angle changes the sensitivity to components of the instantaneous velocity vector. Some recommendations are presented based on long time experiences, e.g. on the choice of probe, on the probe calibration, to the measurement organization and to the evaluation of results.
\end{abstract}

\section{Introduction}

Knowledge of flow behaviour and basic characteristics positively affects development and testing all manner of human work with the fluid flow either outside or inside an object of research, e.g. buildings, jet engines, furnace etc. The significant energy losses are caused by the interactions of boundary layers, wakes, corner vortices etc. generating on immobile or moving parts. Several dynamic phenomena result from these interactions. Apparently the knowledge on the temperature and on the velocity vector distributions in typical flow fields, in several examples of related devices, is contributing to the design and to the improvement of CFD codes. This can accelerate, together, with the knowledge of the flow disturbances features, the development of any device family and also this enables giving a prediction of device's merits and demerits.

A thermo-anemometer is appropriate enough for measurement of the essential individual and joint velocity and temperature characteristics in unsteady nonisothermal 3D-flow field. The first stimulus to start with the development of the procedure were the wish to measure Reynolds shear stress components though only one channel CTA had been available in laboratory. The second stimulus was the suggestion of ŠKODA Turbine establishment to arrange measurements of temperature and velocity turbulent fluctuations in a water steam turbine. Both stimuli encourage to start the solution of the problem at the IT in seventies of twenties century.

It follows from theoretical grounds of thermoanemometry (e.g. Hinze [1]) that basically are both aims achievable [2]. Possibly the oldest executions of velocity, temperature and/or density fluctuations separations from

*jonas@it.cas.cz thermo-anemometer output signal are in connection with Baldwin [3, 4], Corrsin [5,6], Kovasznay [7,8], Morkovin [9] and Sandborn [10,11]. Namely the CCA mode was assumed and the evaluation procedures were complex, originally a semi-graphic evaluation of measurement was applied; Kovasznay [7], introduced "fluctuation diagram" - graphic technique to improve the evaluation of equation with three unknowns, later modified by Morkovin [9] for use in compressible fluid flow. Hajime and Kovasznay [12] demonstrated successfully measurement of Reynolds Stress by a single rotated hot-wire in 1968. Later Hoffmeister [13] published his own rather complex procedure on using a single $\mathrm{HW}$-probe in $3 \mathrm{D}$ turbulent isothermal flow.

Obviously, no new fundamental physical ideas will be presented in the paper but an effective procedure of measurement evaluation and some sources of threatening errors will be described assuming contemporary experiences and devices.

\section{Theoretical considerations and description of the method}

The presented method of measurement by means of a single hot-wire thermo-anemometer is derived on the following assumptions:

1. The investigated object is three-dimensional, non-isothermal and unsteady fluid flow.

2. The fluid properties are close to continuum.

3. The unsteadiness is a superposition of deterministic (particularly periodic) disturbances and statistically stationary random fluctuations of velocity and temperature.

4. The hot-wire probe axis of rotation (direction defined by the unit vector $\overrightarrow{\mathbf{r}}$ ) is fixed and it is 
identical with the positive axis $y$ of the orthogonal coordinate system $(x, y, z)$ introduced in the investigated region (Figure 1) so as to be positive the $x$-component of the mean flow velocity.

5. The wire is inclined to the fixed axis of rotation at the angle $\Theta<\pi / 2$ determined by the scalar product of the unit vectors of the wire direction $\overrightarrow{\mathbf{l}}$ and the axis of rotation $\overrightarrow{\mathbf{r}}$

$$
(\overrightarrow{\mathbf{l}}, \overrightarrow{\mathbf{r}})=\cos \Theta
$$

6. The wire is heated in CTA mode and its cooling in the fluid flow perpendicular to the wire axis is described by the generalized Collis-Williams cooling law

$$
N c=N u\left(\frac{T_{\mathrm{m}}}{T}\right)^{M}=A+B \operatorname{Re}^{N},
$$

together with the relation

$$
\begin{aligned}
& \left(\frac{U(\varphi)}{W}\right)^{2}=\sin ^{2} \varphi+k^{2} \cos ^{2} \varphi=1+\kappa \cos ^{2} \varphi ; \\
& \kappa=k^{2}-1<0 ; 30^{\circ} \leq|\varphi| \leq 150^{\circ},
\end{aligned}
$$

with respect to the concept of Hinze [1] on effective velocity $U$ i.e. heat conduction from a wire skewed to the flow velocity $W$ is equal to the heat convection from the same wire being perpendicular to the "effective" velocity $U$. The angle $\varphi$ is determined by the scalar product

$$
(\overrightarrow{\mathrm{l}}, \overrightarrow{\mathbf{W}} / W)=\cos \varphi
$$

The Nusselt number, $\mathrm{Nu}$ and the wire Reynolds number, $\mathrm{Re}$ are defined as usually e.g. Bruun [14]. Next the notations are introduced: ambient temperature $T(\mathrm{~K})$, hotwire temperature $T_{\mathrm{w}}(\mathrm{K})$ and effective temperature $T_{\mathrm{m}}$

$$
T_{\mathrm{m}}=\left(T_{\mathrm{w}}+T\right) / 2 \text {. }
$$

The calibration parameters $A, B, M, N$ and $\kappa$ must be determined in advance. The best calibration is done in the fluid's state identical at least very similar to the state during experiment. Several other formulations are available on the empirical description of the heat transfer from hot-wire in a fluid flow but here presented seem most universal for gaseous flows.

7. Finally, the assumptions of the thermoanemometer linearized theory are valid.

An example of definitions of the coordinate system and angles notation is shown in Fig. 1 borrowed from [15]. Let us suppose in accord with the Fig. 1 the velocity vector as the superposition of the mean velocity vector and the vector of velocity fluctuations

$$
\vec{W}=\overrightarrow{\bar{W}}+\vec{W} ; \quad|\overrightarrow{\bar{W}}| \gg>|\vec{W}|
$$

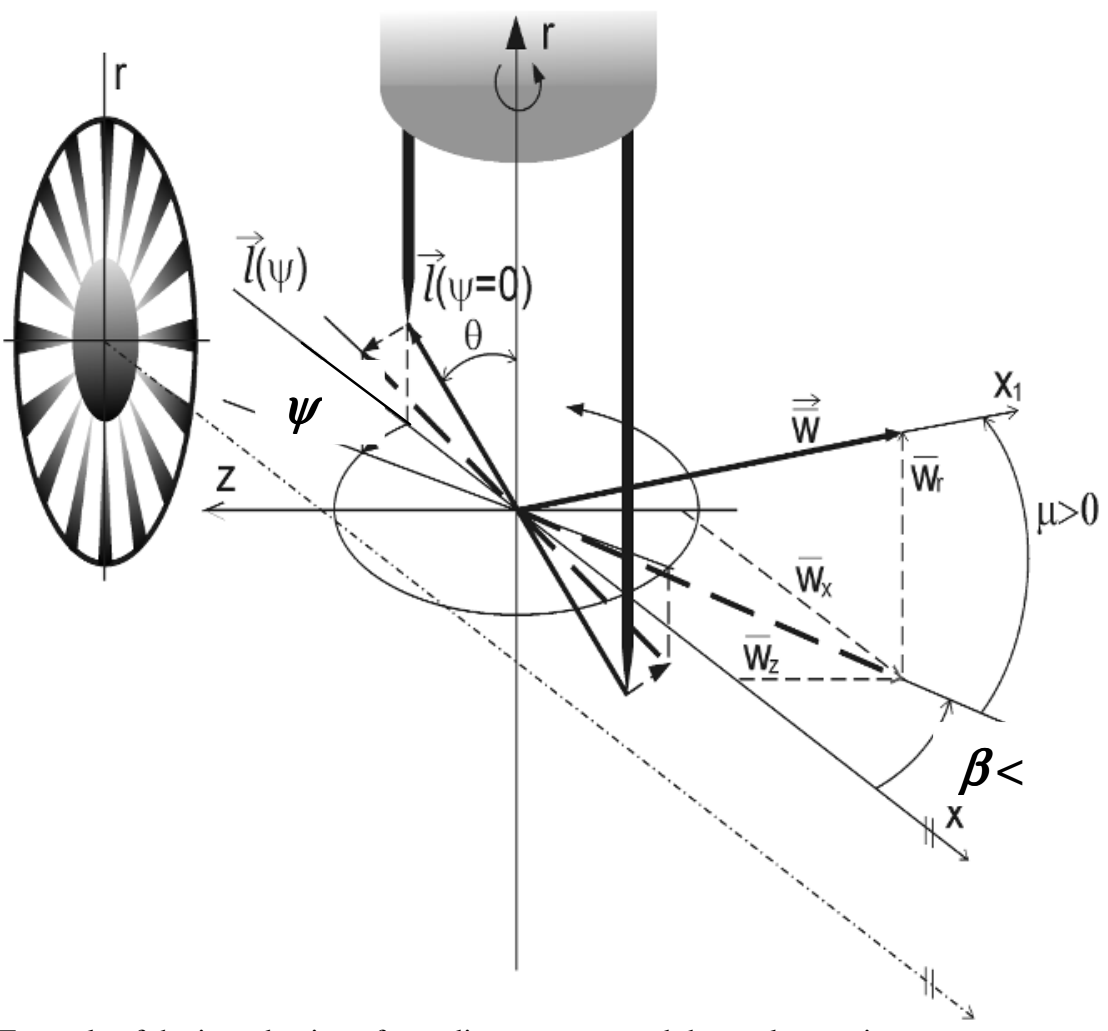

Fig. 1. Example of the introduction of coordinate system and the angle notation in an axisymmetric flow region. 
further the components of the mean velocity vector are

$$
\begin{aligned}
& \bar{W}_{\mathrm{x}}=\bar{W} \cos \beta \cos \mu>0 ; \\
& \bar{W}_{\mathrm{y}}=\bar{W} \sin \mu ; \\
& \bar{W}_{\mathrm{z}}=\bar{W} \sin \beta \cos \mu ;
\end{aligned}
$$

here $\beta$ denotes the pitch angle, angular deflection of the mean velocity vector from the plane $(x, y) \equiv(x, r)$ and $\mu$ is the yaw angle, angular deflection of the mean velocity vector from the plane $y \equiv r=$ const.

The wire starting position $\psi=0$ is in the plane $(x, y) \equiv$ $(x, r)$ with the shorter prong upstream the longer one then the components of the wire direction unit vector are

$$
\psi=0 \rightarrow l_{x}=-\sin \Theta, l_{y} \equiv l_{r}=\cos \Theta, l_{z}=0 .
$$

Turning the wire of angle $\psi$ (clockwise positive angle) from the starting position with the wire in the plane $(x, r)$ then the components of the wire direction unit vector become

$$
\begin{aligned}
& \psi \neq 0 \rightarrow l_{\mathrm{x}}=-\sin \Theta \cos \psi, \\
& l_{\mathrm{y}} \equiv l_{\mathrm{r}}=\cos \Theta \\
& l_{\mathrm{z}}=-\sin \Theta \sin \psi .
\end{aligned}
$$

Next the angle between the wire and the mean velocity $\varphi$, the wire setting angle, can be evaluated from the scalar product (4) and substituted into the directional sensitivity equation (3). Then after some formal rearrangement the formulae (3) is rewritten

$$
\begin{aligned}
& (\bar{U} / \bar{W})^{2}=1+ \\
& +\kappa[\cos \Theta \sin \mu-\sin \Theta \cos \mu \cos (\psi-\beta)]^{2}, \\
& \mu \neq \pi / 2 .
\end{aligned}
$$

From this it is clear that the left side of this relation equals maximum if

$$
\begin{aligned}
& \cos \left(\psi_{\mathrm{a}}-\beta\right)=\cos \left(\psi_{\mathrm{b}}-\beta\right)=\operatorname{tg} \mu / \operatorname{tg} \Theta, \\
& \psi_{\mathrm{b}}=2(\pi+\beta)-\psi_{\mathrm{a}},
\end{aligned}
$$

here $\psi_{\mathrm{a}}$ and $\psi_{\mathrm{b}}$ are turning angles at which the wire is perpendicular to the mean velocity i.e. position of maximal wire heat loss that appears in maxima of the CTA output signal $\bar{E}_{\mathrm{a}}, \bar{E}_{\mathrm{b}}$. The relation is valid

$$
\psi_{\mathrm{b}}=2(\pi+\beta)-\psi_{\mathrm{a}}
$$

The left side of the relation (10) equals minimum if

$$
\sin \left(\psi_{\mathrm{c}}-\beta\right)=\sin \left(\psi_{\mathrm{d}}-\beta\right)=0 ; \quad \psi_{\mathrm{c}}=\pi+\beta
$$

Two minima of the wire heat loss appear with $\bar{E}_{\mathrm{c}}$ and $\bar{E}_{\mathrm{d}} \ll \bar{E}_{\mathrm{c}}$ at turning angles $\psi_{\mathrm{c}}$ and $\psi_{\mathrm{d}}$. The physical significance has the angle $\psi_{\mathrm{c}}$ at the probe position with the shorter prong/electrode upstream the longer one. Opposite direction $\psi_{\mathrm{d}}$ corresponds to the hot wire position in the longer prong wake. Then a deeper decrease of the output signal $\bar{E}$ comes that is accompanied by an extensive amplification of the fluctuating component e.g. $\sqrt{\overline{e^{2}}}$. Obviously the relation must valid

$$
\psi_{\mathrm{c}}=\left(\psi_{\mathrm{a}}+\psi_{\mathrm{b}}\right) / 2
$$

Having in mind the relations (11), (13) and (14), the direction of the mean velocity vector could be determined simply from the measurement of the CTA output signal distribution $\bar{E}$ versus $\psi$ and evaluating extremes. Of course, the wire temperature and the fluid flow parameters must be unchanged during measurement of the distribution $\bar{E}(\psi)$. The modulus of the mean velocity vector $\bar{W}$ is then calculated using the cooling law (2). Then it is possible to check the derived transformation of the mean output signal distribution into the relevant distribution of the normalized effective velocity. The equation with the empirical coefficient $\kappa$ must valid

$$
\bar{E}(\varphi(\psi)) \rightarrow\left(\frac{\bar{U}}{\bar{W}}\right)^{2}=1+\kappa \cos ^{2} \varphi
$$

Interpretation of the output signal fluctuations follows from the cooling law (2) and the equation (3) of the directional sensitivity of a heated wire. In the framework of the linearized thermo-anemometer theory are valid formulas

$$
\begin{aligned}
& \frac{e}{\bar{E}}=F \frac{u}{\bar{U}}+G \frac{t}{\bar{T}}, \\
& \frac{u}{\bar{U}}=F_{1} \frac{w_{1}}{\bar{W}}+F_{2} \frac{w_{2}}{\bar{W}}+F_{3} \frac{w_{3}}{\bar{W}} .
\end{aligned}
$$

The sensitivities to the effective velocity fluctuations and to the temperature fluctuations are derived in the forms ${ }^{1}$

$$
\begin{aligned}
& F=\frac{N}{2} \frac{\mathrm{Nc}-A}{\mathrm{Nc}} \\
& G=-\frac{1}{2}\left[\begin{array}{l}
\frac{\bar{T}}{T_{\mathrm{w}}-\bar{T}}- \\
\left.-\frac{\bar{T}}{T_{\mathrm{w}}+\bar{T}}\left(n_{\lambda}+M \frac{T_{\mathrm{w}}}{\bar{T}}-2\left(n_{\mu}-n_{\rho}\right) F\right)\right] .
\end{array}\right.
\end{aligned}
$$

The partial derivatives $F_{i}, \quad i=1,2,3$ are better transparent if the mean flow coordinate system is introduced.

\footnotetext{
${ }^{1}$ Heat conductivity, dynamic viscosity and density in gas flow are assumed in forms:$$
\lambda=k_{\lambda} T^{n_{\lambda}} ; \mu=k_{\mu} T^{n_{\mu}} ; \rho=k_{\rho} P T^{n_{\rho}} .
$$ 


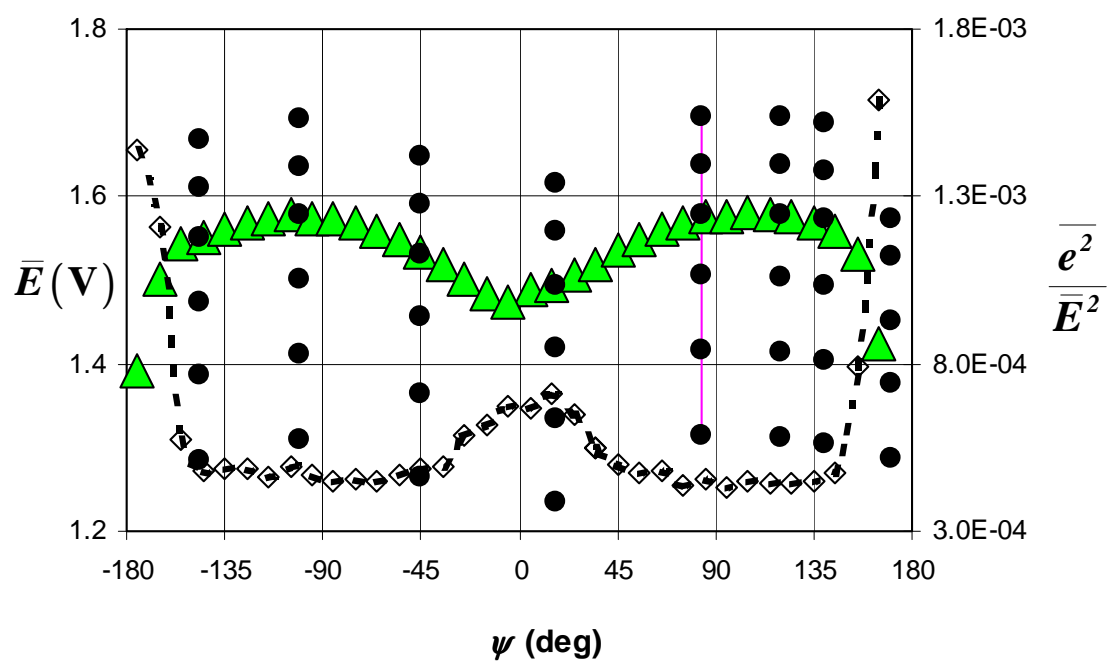

Fig. 2. Distribution of the CTA output signal mean value and its dimensionless variance (triangles $T_{\mathrm{w}}=225^{\circ} \mathrm{C}$, circles $T_{\mathrm{w}}=150^{\circ}, 275^{\circ} \mathrm{C}$, rhombus variance $e$ ).

The mean flow system $\left(\mathrm{x}_{1}, \mathrm{x}_{2}, \mathrm{x}_{3}\right)$ is defined by the conditions

$$
\overrightarrow{\vec{W}}=\left[\bar{W}_{1}=\bar{W}, \bar{W}_{2}=0, \bar{W}_{3}=0\right] .
$$

It is easy to transform the coordinate system $(x, y, z)$ into $\left(x_{1}, x_{2}, x_{3}\right)$ e.g. the components of the HW unit vector (9) become form

$$
\begin{aligned}
& l_{1}=-\sin \Theta \cos \mu \cos \psi+\cos \Theta \sin \mu, \\
& l_{2}=\sin \Theta \sin \mu \cos \psi+\cos \Theta \cos \mu, \\
& l_{3}=\sin \Theta \sin \psi .
\end{aligned}
$$

Then the partial derivatives $F_{\mathrm{i}}, \quad \mathrm{i}=1,2,3 \mathrm{read}$

$$
F_{1}=1, F_{2}=\frac{\kappa l_{1} l_{2}}{1+\kappa l_{1}^{2}}, F_{3}=\frac{\kappa l_{1} l_{3}}{1+\kappa l_{1}^{2}} .
$$

The evaluation of the dimensionless variances of the effective velocity fluctuations and the temperature one together with the dimensionless covariance of velocity and temperature consists in the interpolation of the linear function with three unknowns

$$
\left(\frac{\overline{e^{2}}}{\bar{E}^{2}}\right)_{\substack{\mathrm{T}_{\mathrm{w}} \\ \psi}}=\left(G^{2} \frac{\overline{t^{2}}}{\bar{T}^{2}}+2 F G \frac{\overline{t u}}{\bar{T} \bar{U}}+F^{2} \frac{\overline{u^{2}}}{\bar{U}^{2}}\right)_{\substack{\mathrm{T}_{\mathrm{w}} \\ \psi}},
$$

subscripts at the round brackets indicate that measurement of $\overline{e^{2}} / \bar{E}^{2}$ must be done at several wire temperatures $T_{\mathrm{w}}$ and some roll angels $\psi$.

Only afterwards they are possible the interpolations of the turbulent heat transfer vector components

$$
\overline{w_{\mathrm{i}} t} /(\bar{W} \bar{T}), \mathrm{i}=1,2,3
$$

by means of the formulae

$$
\left(\frac{\overline{u t}}{\bar{U} \bar{T}}\right)_{\psi}=F_{1}(\psi) \frac{\overline{w_{1} t}}{\bar{W} \bar{T}}+F_{2}(\psi) \frac{\overline{w_{2} t}}{\bar{W} \bar{T}}+F_{3}(\psi) \frac{\overline{w_{3} t}}{\bar{W} \bar{T}}
$$

and the evaluation of the dimensionless Reynolds stress tensor components $\overline{w_{\mathrm{i}} w_{\mathrm{j}}} / \bar{W}^{2}$ requires the interpolation of the function with six unknowns

$$
\left(\frac{\overline{u^{2}}}{\bar{U}^{2}}\right)_{\psi}=F_{\mathrm{i}}(\psi) F_{\mathrm{j}}(\psi) \frac{\overline{w_{\mathrm{i}} w_{\mathrm{j}}}}{\bar{W}^{2}} ; \mathrm{i}, \mathrm{j}=1,2,3
$$

\section{Practical application of the rotary slanted single wire CTA}

As an example the distribution of the output voltage from CTA $\bar{E}$ with wire heated on $225^{\circ} \mathrm{C}$ is shown in Fig. 2 (triangles) together with walues of $\bar{E}$ measured at wire temperatures $\mathrm{T}_{\mathrm{w}}$ between $150^{\circ} \mathrm{C}$ and $275^{\circ} \mathrm{C}$ (black circles). Distribution od the normalized variance of the signal fluctuations demonstrates the difference in the fluctuations level with the wire in wake of longer prong and with the wire upstream the longer prong.

An agreement of the mean flow measurement results and the calibration of the wire directional sensitivity is demonstrated in Fig. 3. The evaluated ratio of the mean effective velocity and the mean flow velocity is plotted against the formulae (10) with the empirical coefficient $\kappa$ (evaluated from the directional sensitivity calibration). The distribution in Fig. 3 is the certification that the evaluations of the mean flow characteristics are right. 


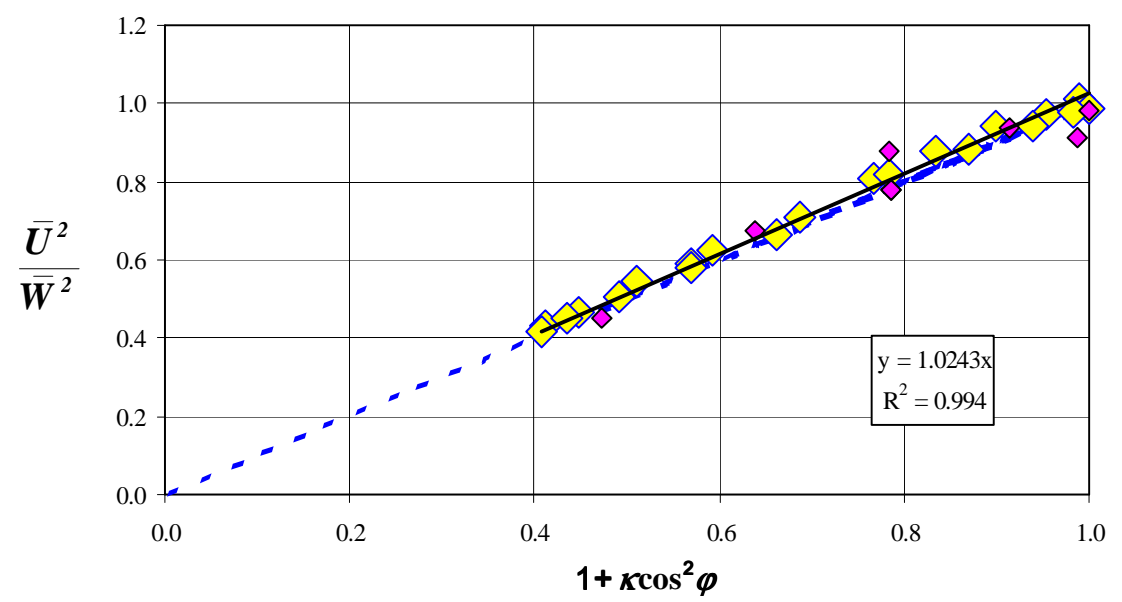

Fig. 3. Comparison of the measurement with the directional sensitivity calibration.

A simple check of the statistical estimates of the dimensionless variances of the effective velocity fluctuations and the temperature fluctuations and of the dimensionless covariance of velocity and temperature is shown in Figure 4. The interpolated value of the nondimensional variance $\overline{e^{2}} / \bar{E}^{2}$ is evaluated by substituting statistical estimates

$\frac{\overline{t^{2}}}{\bar{T}^{2}}, \frac{\overline{t u}}{\bar{T} \bar{U}}, \frac{\overline{u^{2}}}{\bar{U}^{2}}$

into the equation (21). Comparison with the value following at the same conditions from measurement illustrates the accuracy of interpolation.

For the discussed example the ratio interpolation divided by measurement equals $(1 \pm 0.08)$ though estimates of the relative errors $\overline{t^{2}}$ and $\overline{t u}$ are very high 50 and 80 percent; the estimated error of $\overline{u^{2}}$ is 5 percent. It should be noticed that the presented example was selected from an industrial measurement. At that time the flow was without important sources of heat flux and virtually turbulent heat transfer was negligible.

The statistical estimates of the dimensionless Reynolds stress tensor components $\overline{w_{i} w_{j}} / \bar{W}^{2}$ determine the function (24) i.e. the interpolation of the distribution $\overline{u^{2}} / \bar{U}^{2}$ versus the roll angle $\psi$. Their evaluation is conditioned by additional requirements derived from laws of mathematics and physics.

The Reynolds stress tensor components are

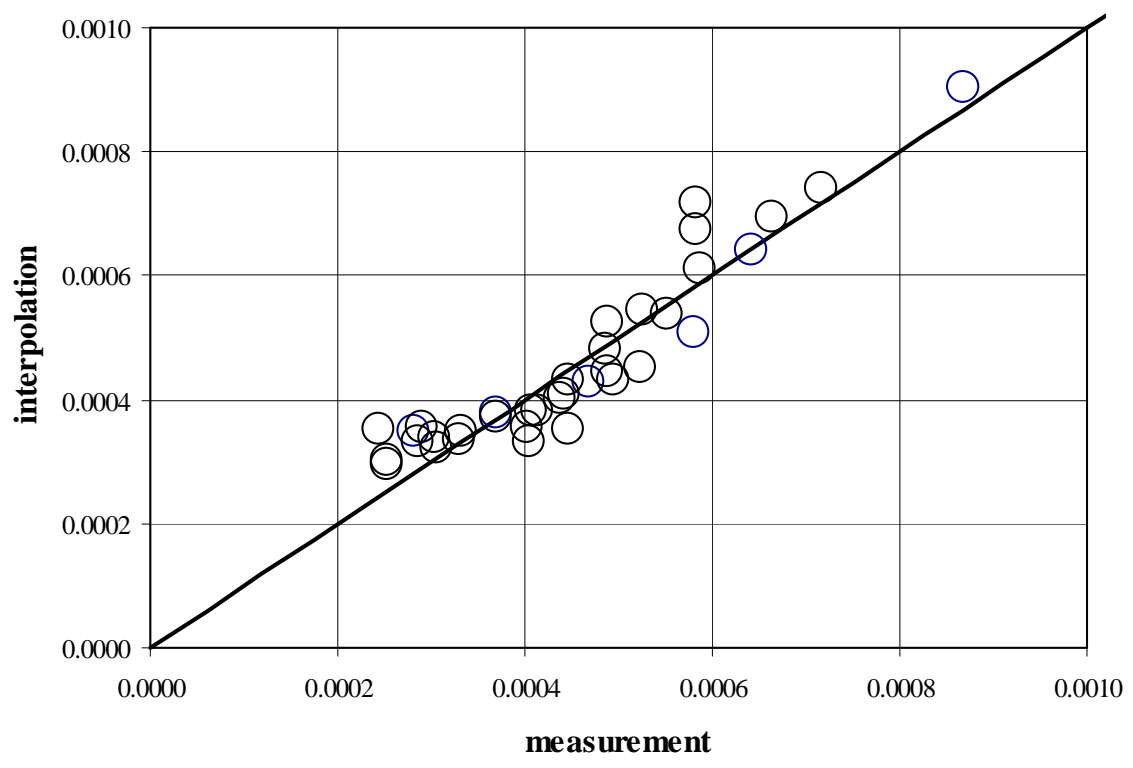

Fig. 4. Comparison of the measured dimensionless output signal variance $\overline{e^{2}} / \bar{E}^{2}$ with the interpolation (21). 


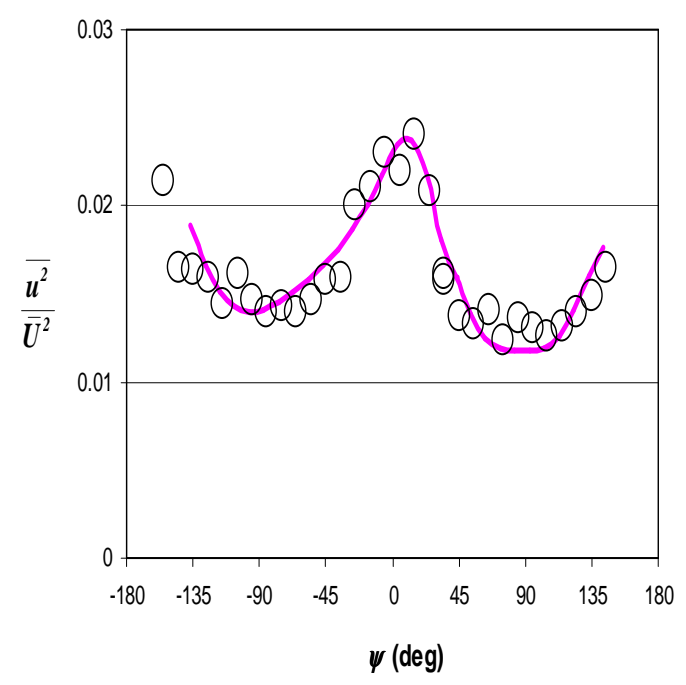

Fig. 5. Interpolation of the dimensionless variance of effective velocity fluctuations.

conditioned by additional requirements namely the diagonal components must be positive and the absolute value of correlation coefficients must reach greatly one

$$
\overline{w_{\mathrm{i}}^{2}}>0, \quad \overline{w_{\mathrm{i}} w_{\mathrm{j}}} / \sqrt{\overline{w_{\mathrm{i}}^{2} w_{\mathrm{j}}^{2}}} \leq 1, \quad \mathrm{i} \neq \mathrm{j}
$$

The comparison of measurement results (circles) and the interpolation (full line) is shown in Fig. 5. However the measurement's scatter seems big, the calculated relative probable error of the interpolation is only 6 percent.

The presented example is describing a non isothermal flow of the overheated water steam in an industrial device with turbulence level 11.6 percent and with the intensity of temperature fluctuations 1.9 percent. Some other examples in the high speed air flow and flow of a wet water steam might be found in references [15 - 19].

\section{Conclusions}

The useful tool is presented for the experimental investigations of complex three dimensional nonisothermal unsteady fluid flows fulfilling exactly determined assumptions that are only little restricting the employment.

The single slanted heated wire, rotary round the fixed axis, heated by a CTA on several operating temperatures constitutes this tool.

Theoretical grounds of the procedure are illustrated by an example from some industrial measurement. More neatly arranged results are received from laboratory experiments.

\section{Acknowledgements}

This work has been supported by the Czech Science Foundation, the project GA101/08/1112 and
GAP101/12/1271 and with the institutional support, projects AV0Z20760514.

\section{References}

1. J.O: Hinze, Turbulence - an introduction to it mechanism and theory (Mc-Graw Hill, New York, 1959)

2. P. Jonáš, Strojnický časopis XXIV, No. 2-3, 110-126 (1973)

3. L.V. Baldwin, V.A. Sandborn, and J.C. Laurence, Paper No. H-149-58, ASME, (1958)

4. L.V. Baldwin, NACA TN 4369, (1958)

5. S. Corrsin, Rev. Sci. Instr., 18, 469-471 (1947)

6. S. Corrsin, Turbulence: Experimental methods. In Handbuch der Physik, Vol. 8.2 (Springer, Berlin, 523-590 (1963)

7. L.S.G. Kovasznay, J. Aero- Sci. 17, 9 (1950)

8. L.S.G. Kovasznay, J. Aero- Sci. 20, 10 (1953)

9. M.V. Morkovin, AGARDograph 24 (1956)

10. V.A. Sandborn, Metrology Press, Fort Collis, Colorado (1972)

11. V.A. Sandborn, Advances in hot -wire anemometry (Eds. W-L- Melnik and J.R. Weske, University of Maryland, Rep. AFOSR No. 68-1492 1968)

12. Hajime Fujita, L.S.G. Kovasznay, Rev. of Sci. Instr., 39, 1351-1355 (1968)

13. M. Hoffmeister, DISA Information, No. 13, 26-29 (1972)

14. H.H. Bruun, Hot-wire anemometry (Oxford University Press, Oxford 1995)

15. P. Jonáš, O. Mazur and V. Uruba, Proc. $6^{\text {th }}$ Eur. Conf. on turbomachinery (Eds. Bois G., Sieverding C., Manna M. and Arts T., Vol. 1, Lille, France, March 7-11, 480-489, 2005)

16. P. Jonáš, Hochschulkurs Hitzdrahtanemometrie (Ed. R. Ermshaus , Uni. Karlsruhe, October 4-6, Pt. 9, 1995)

17. Jonáš P., Mazur O. and Uruba V., Proc. $4^{\text {th }}$ Eur. Conf. on turbomachinery (Eds. Bois, G., Decuypere, R.; Martelli, F. March 20-23, Firenze, Italy, 833-842, 2001)

18. P. Jonáš, O. Mazur, V. Řehák and V. Uruba, Transactions of the Inst. Fluid-Flow Machinery, 43 No.113, Gdansk, 191-200 (2003)

19. V. Uruba, P. Jonáš, O. Mazur and V. Řehák, Proc. $5^{\text {th }}$ Eur. Conf. on turbomachinery (Eds. Št'astný, M.; Sieverding, C. H.; Bois, G., Praha, March 17-22, Czech Republic, 709-718 2003). 